\title{
Service System Analytics: Cost Prediction
}

\author{
Wolfgang Seiringer ${ }^{1}$, Jorge Cardoso ${ }^{2,3}$, and Johannes Kunze von Bischhoffshausen ${ }^{2}$ \\ ${ }^{1}$ Institute of Software Technology and Interactive Systems \\ Vienna University of Technology \\ Favoritenstrasse 9-11, Vienna, Austria \\ wolfgang@seiringer.info \\ ${ }^{2}$ Karlsruhe Service Research Institute \\ Karlsruhe Institute of Technology, Karlsruhe, Germany \\ jorge.cardoso@kit.edu, johannes.kunze@kit.edu \\ ${ }^{3}$ CISUC/Departamento de Engenharia Informática \\ Universidade da Coimbra, Coimbra, Portugal \\ jcardoso@dei.uc.pt
}

\begin{abstract}
Services become every day more important for society. Cost prediction is one important aspect which has not received the required attention. Nonetheless, its impact on the economic success of service-based industries is considerable and, so, it cannot be overlooked. Therefore, in this paper we tackle the problem of cost prediction of service systems. Since previous work generally followed an ad hoc approach, we present a three layered approach based on customer factors to measure customer involvement since it is a good predictor for service cost. Service co-creation between customer and provider is the central concept of our costing approach. For service modelling we use Linked USDL, and to reason on costing data we use methods from service analytics. On the one hand, we reduce the uncertainty introduced by customers and can predict more accurate service costs. On the other hand, the systematic approach enables a better understanding of the costing problem and the service systems under study, and increases reusability of service analytics algorithms.
\end{abstract}

Keywords: Service cost modelling, service analytics, Linked USDL, cocreation, customer factors, service system, customer involvement, service modelling, business analytics.

\section{Introduction}

During service provisioning, provider and customer are part of a service system to cocreate a service. The process of co-creation requires both partners to invest resources (e.g. human resources, knowledge, and energy). Co-Creation is a concept which is not limited to Business-to-Business (B2B), it can also be observed in the Business-toConsumer (B2C) context. In B2B and B2C both partners participate and invest resources into co-creation. Service provisioning also requires a provider to integrate and account for providers' human resources and customer factors (CF). Customer factors are all the tangible and intangible aspects which influence co-creation and can only be provided by the customer. Examples of customer factors include information, 
knowledge, experience, and commitment that customers bring in during the cocreation. For a provider, customer factors are a source of uncertainty since they affect service performance and service costs [2]. To reduce the gap between forecasted and real service costs, the modelling and understanding of customer factors and their impact on costs is fundamental. This will increase the precision of service cost modelling.

We propose an approach to extend established service costing methods with customer factors. The approach requires modelling the concept of service systems to facilitate inferring service costs by means of service analytics. We model service systems using the Linked Unified Service Description Language (Linked USDL). Since the consumed time of a resource can be used as a proxy for cost, we will rely on the process model of USDL to calculate the required time to complete a service. In other words, USDL enables to build a process model of a service which can then be used for service costing.

To achieve this goal, an extension of the business process is necessary to integrate customer factors on service cost. In our approach, we extend processes by adding a new view for costing in the form of a function to measure a customer's individual influence on service performance. For each identified customer attribute a function is constructed and mapped on the customer factors. USDL serves as a service model which provides a schema that enables methods from the field of service analytics to derive cost information. As a result of our study, we concluded that Linked USDL provides a suitable logical framework to reason of services' characteristics such as cost. With our research we want to find answers to the following questions: Does a systematic approach yields a greater precision for service-based cost modelling? Can the element of co-creation and customer factors yield better models for cost modelling? We claim that a systematic approach 1) including customer factors, 2) using service schema/instance modelled with USDL, and 3) services analytics methods can provide a sound solution for service-based cost modelling.

Service costing and service analytics have a direct impact on the competitiveness of organizations and on the reinvigoration of service-based economies. A better estimation of the cost of services based on customer factors leads to more fit service offerings.

This paper is organized as follows. In section two we describe a maintenance service to motivate our proposed approach. Section three introduces related work. Afterwards in section four the concept of customer factors is presented. In section five we write about service modelling and show how customer factors can be integrated into Linked USDL. In the following section six we describe concepts of service analytics and finally we conclude in section seven.

\section{Motivation Use Case}

Our use case is based on a world-wide offered B2B maintenance service of a medium-size manufacturing company producing medical devices for laboratories. Cocreation starts when the customer contacts the provider since maintenance is due. We divide co-creation into provider and customer domains and analyse service activities which (1) are required for co-creation (2) and with a direct relation to the offered 
service. To co-create maintenance the provider has to perform all or only selected service activities. This means which business partner is responsible for, which service activity and, which service activities have to be performed, is defined and documented before co-creation. To document this kind of commitment the usage of a Service-Level Agreement (SLA) is a possibility. In our case identified service activities are check device status, send spare parts to customer, send technician to customer, install spare parts, update device software and teach the usage of new functions. To account for a provider's service costs, it is necessary to measure the cost effects on service performance during co-creation. For example to co-create service activity check device status the provider's performance, and activity time consumption, relies on a problem description submitted by an employee of the customer. Because the maintenance costs vary for each customer the provider requires a flexible costing approach. Furthermore, instead of having fixed costs for the maintenance service, the provider wants also to include customer depending factors.

\section{Related Work}

Activity-Based Costing ( $\mathrm{ABC}$ ) is a well-known approach to account service costs. $\mathrm{ABC}$ has its origin in the manufacturing industry [3]. A further development of $\mathrm{ABC}$ is Time-Driven Activity-Based Costing (TDABC). Here time is regarded as the leading cost driver. Because most of the supplied and consumed resources like employees and machines can be measured using time. In contrast to $A B C$, the approach of TDABC is easier to maintain and to keep the used costing data up to date [4]. In production theory a similar concept, compared to our customer factors, exists based on production factors and factor combination [5]. Production factors are split up in internal and external factors. A production factor can be, for example, a defective medical device, information, or the customer himself. The internal factors are entities that are provided by the producer. The customer supplies the external factors. It is not possible for the provider to produce or buy external factors on the market. Also the associated knowledge is under control of the service customer. The producer is forced to integrate it into the production process. Factor combination means that internal and external factors are combined to obtain the desired product. This implies that both sides have to provide resources during co-creation [6].

In contrast to $\mathrm{ABC}$ and $\mathrm{TDABC}$ we also consider customer introduced uncertainty. Compared to production factors, we present a concept extending to the customer domain, called customer factors.

\section{Co-Creation and Customer Factors}

We use the developed concept of customer factors to quantify the uncertainty introduced by a customer and rely on TDABC to compute service costs. Standardisation of service processes is difficult due to the heterogenic nature of services [7]. Because of heterogeneity, customer factors vary in quantity and quality. From a practical perspective it is impossible to model all the changing customer factors. Our research work is focused to measure the impacts on service performance and not the kind of individual customer factors. 
To understand the monetary impact of customer factors we made a literature review with a focus on the following three aspects. (1) Is the exchange of resources associated with co-creation? (2) Are customer factors used to calculate services costs? (3) Which kind of customer attributes are used to describe customer factors? We can summarize that there is a common understanding about a necessary resource exchange between service provider and service customer. We could not find specialized methods trying to measure the impact of customer involvement on service costs using customer factors. In all selected articles we could find a, detailed or at least a vague description about the, correlation between customer involvement and costs. The kind of stated customer factors or customer attributes range from not precisely specified to concrete values [8], e.g. health care information [9], project complexity, company size, experience [10], commitment, information [11], cocreation activities [12] or knowledge about customer profiles [13]. Based on this input we argue that each customer provides individual customer attributes and it is necessary to consider them for performance measurement. We have derived four abstract customer factors: co-creation, information, technology and experience. They are conceptualized to measure the impact on activity time consumption. Subsequently cost can be calculated based on activity time using the associated resource cost e.g. the salaries of employees. We have defined customer factors which are easy to associate with customer related influence on co-creation process. From previous publications, e.g. [14], we know that a clear semantic of customer factors supports applying a costing method designed for services. Using individual customer attributes to describe the general concept of customer factors requires a generalized approach and stable mapping from attributes to factors and from factors to resources. Metrics for customer attributes must be defined for each service case individually. This procedure enables our approach also to provide reasonable values for changing and difficult to quantify customer input. The discipline of service analytics provides techniques supporting the identification and calculation of service attributes. We use three abstraction levels to map the concept of customer factors to service costs. The first level is used to capture customer individual attributes like company size or number of employees and map them to our four customer factors. Customer factors constitute the second level. The mapping of a customer attribute to a customer factor has to be decided individually and is based on the expressed semantic meaning. For example number of employees can be associated with information and capacity of internet connection with technology. For the third level, Time-Driven Activity-Based Costing, customer factors are then mapped to the concept of TABC. Customer attributes are not directly mapped to TABC to have a stable relation between customer factors and their impacts on service costs. We have selected the following four customer factors because (1) they provide a comprehensive selection, (2) their intention is clear, (3) they are applicable to different service cases and (4) are able to capture the complete range of possible customer attributes and service characteristics. The $\mathrm{CF}$ are:

Co-Creation $\left(C_{i}\right)$ : Describes the level of co-creation. A high co-creation level indicates a high level of customer involvement. The more activities are performed by the customer the higher the level of co-creation. Co-creation is the ratio between the number of involved service activities and the number of service activities performed by the customer. Compare also (2) where the first term is used to calculate $C_{i}$.

Information $\left(I_{i}\right)$ : For a provider, $I_{i}$ measures the degree of dependency on external information. An example customer attribute from our maintenance scenario mapped to $I_{i}$ is the average number of service activities per co-creation. 
Technology $\left(T_{i}\right)$ : Indicates the importance of technology during co-creation e.g. infrastructure capabilities or IT service availability. From our service case we can map the average number of e-mails to $T_{i}$.

Experience $\left(E_{i}\right)$ : Indicates the degree of experience and competencies that are required by the involved human resources in co-creation. We can map the time being in relation with a customer or number of maintenance co-creations to $E_{i}$.

All four customer factors are applied on an individual activity $A_{i}$, see (1). Variables $I_{w}, T_{w}$ and $E_{w}$ are used to weight the impact of these three customer factors and enable weighting varying importance of customer factors. Please note that the weight of the customer factors can be different. Only the sum must be 1 to get a normalized value, which is subsequently comparable between service cases and customers.

$$
C F_{A i}=I_{i} * I_{w}+T_{i} * T_{w}+E_{i} * E_{w}, \text { with }\left(I_{w}+T_{w}+E_{w}\right)=1
$$

To get individual customer factor values $\left(I_{i}, T_{i}\right.$ and $\left.E_{i}\right)$ we use the formula e.g. $I_{i}=\frac{\sum_{j=1}^{m} C A_{i}}{m}$ where $C A_{i}$ are customer attributes and $m$ is the different number of $C A_{i}$ mapped to $I_{i}$. To map a customer attribute to a customer factor we use the injective function $f: C A_{i} \rightarrow C F_{A i}$. The output of our service model for service $S_{i}$ is a parameter value indicating the impact of customer involvement during co-creation, compare (2). Where $C F_{S_{i}} \in R$ and only activities $A_{i}$ are including where $C_{i}$ is 1 . The value of $C_{i}$ can be 0 (not an involved service activity) or 1 (is an involved service activity). The usage of $C_{i}$ allows calculating individual service costing scenarios for different customers. To increase the importance of an individual customer attribute, it is also possible to weight the individual $C A_{i}$ like described for $C F_{A i}$. For this paper we have selected the average method to calculate a single customer attribute, which has no negative consequences to the semantic of this concept.

$$
C F_{S_{i}}=\frac{\sum_{i=1}^{n} C_{i}}{n}+\frac{\sum_{i=1}^{n} C F_{A_{i}}}{n}, n \text { is the number of service activities }
$$

\section{$5 \quad$ Service Modeling}

So far, current approaches for service analytics look into developing algorithms to provide insight on, e.g., cost estimation and service performance. Nonetheless, the object of analysis, the service system, is not conceptualized. This leads often to the development of algorithms which are hard to reuse since they are developed to analyze particular services. Therefore, we propose and rely on the adoption of an abstraction by using a "master schema" for representing services. For our work, we use Linked USDL (the Universal Service Description Language, www.linkedusdl.org), one of the most comprehensive service models developed up to date when compared to WSDL, OWL-S, SAWSDL, hREST, MicroWSMO, WSMO-lite, or WSMO [15].

Considerable improvements were made to technology with the introduction of Service-Oriented Architecture (SOA) concept. Nonetheless, one of the main limitations is that service-orientation was introduced to solve integrations problems. SOA was biased by an enterprise-centric vision. Linked USDL was developed as an answer to this partial view by bridging a business, an operational and a technical 
perspective on services. The language models service concepts and properties such as service level, pricing, legal aspects, participants, marketing material, distribution channels, bundling, operations, interfaces, resources, etc. Linked USDL is a language which has evolved from USDL [15] by using a semantic representation for the schema.

\subsection{Linked USDL and Service Costing}

Linked USDL provides a wide range of concepts which can be used for costing. These are organized in 4 modules: core, pricing, service level, and legal. In this paper we will only use the core module to provide illustrative examples, namely:

- usdl:service \& usdl: InteractionPoints. A service is described by a set of interaction points. Interaction points have been introduced in the context of service design and blueprinting [16] and have gained a wide adoption in service marketing and operations management. Each service activity from our scenario has a set of interaction points. Using Linked USDL, these points are identified and represented with USDL.

- usdl:InterationType. An interaction is characterised with a mode (manual, semi-automated, human-machine, machine-human, or automated) and a space (on-site or remote). Both, the mode and the space, provide additional information on an interaction which enable a better cost estimation.

- usdl:receives \& usdl:yields. An interaction involves the exchange of resources (e.g. information. documents, knowledge, etc.). The concept receives captures the resources needed for an interaction to take place, while the concept yields describes the resources that are generated.

During service provisioning, information pertaining these concepts is captured and integrated. It constitutes a base for a systematic service costing and service analytics.

\subsection{Questions Answered by Linked USDL}

The Linked USDL can "answer" to various competency questions [17]. For example, "which participants were involved during the interaction points of the service activity process customer inquiry". These can be seen as primitives that service analytics can rely on to identify hidden patterns associated with cost. In this section, we illustrate how they are related to service costing. Examples were selected to answer to three types of questions (identified with CQ1-3) which involve the concept usdl: InterationPoints: who, how, and what.

- Who (CQ1). Who was involved during the provisioning of a service or a particular interaction point? An answer to this question provides information for the $\mathrm{CF}$ co-creation $C_{i}$ and $\mathrm{CF}$ experience $E_{i}$. Two types of information can be obtained. On the one hand, the role of a business entity involved (e.g., customer, provider, observer, or third party supplier) and, on the other hand, the role taken when participating in the interaction. For example, if the interaction role was 'observer', then the impact of the customer on co-creation and service cost would be lower than if its role would have been an active 'participant'. 
- How (CQ2). How was the interaction conducted? The degree of automation of an interaction (e.g., manual, semi-automated, or automated) is related to service cost, but the relationship is not always trivial. An answer to this question provides information for the CF co-creation $C_{i}$ and CF technology $T_{i}$. .

- What (CQ3). What resources were used during interactions? The use of more or less resources influence the cost and complexity of an interaction. Therefore, resources need to be an integral part of a $\mathrm{CF}$ evaluation function. An answer to this question provides information for the CF information $I_{i}$, CF technology $T_{i}$ and $\mathrm{CF}$ experience $E_{i \text {. }}$

It should be noticed that the variation of the cost and customer factors associated with the situations described by these questions is context (service, customer) dependent.

\subsection{Using Linked USDL for Service Analytics}

Once Linked USDL is populated with instances which represent the provisioning of services, it can be queried by algorithms from the field of service analytics. In this section, we will provide an explanation of the underlying idea. The following example illustrates the application of competency question CQ1 to identify the roles of the stakeholder (i.e. entities) who were involved during the provisioning of the service Maintenance by executing the various activities of the associated process (i.e. from check device status to teach the usage of new functions) from our motivation use case (see Section 2). We use SPARQL to define queries since this is the language commonly used to query RDF structures, the underlying representation format of Linked USDL.

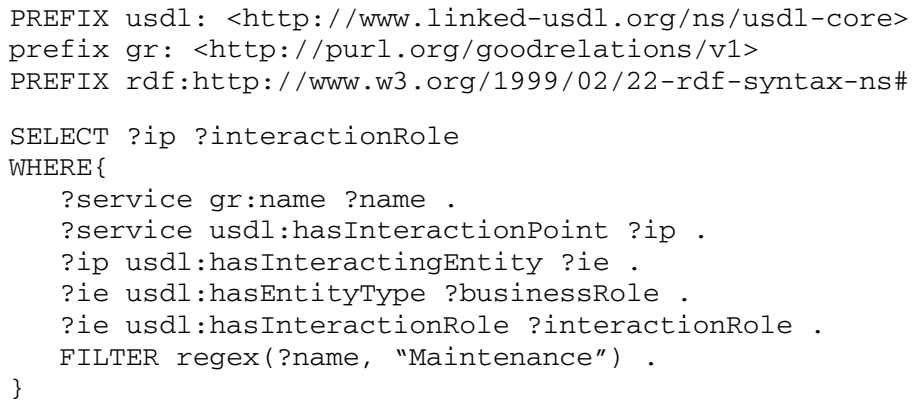

The query starts by identifying all the interaction points executed during the provisioning of the service Maintenance. Afterwards, for each interaction associated with, e.g. checking devices status or sending spare parts to customer, the query identifies the business roles of the stakeholders involved using the variable ?businessRole (which can take the form of a regulator, a producer, an intermediary, etc.) and the interaction role involved using the variable ? interactionRole (e.g. an observer, a participant, an initiator, a mediator, etc.). This information about who was involved during the realization of interactions points is then used by algorithms from the field of service analytics, e.g. clustering and classification algorithms, to extract new knowledge which enable to create better cost estimates. 


\section{Service Analytics}

Within the process of co-creation, where both partners bring in their knowledge, skills, and resources, both partners form a service system [1]. For realizing service analytics for cost accounting, there is a need to capture, process, and analyze data within such a service system. This application where analytics supports services systems has been proposed as services analytics [18]. Service analytics can be decomposed along two different dimensions. The first dimension distinguishes the different types of data sources, whether the data was tracked within the provider organization, at the customer side, or during the encounter between both partners. Therefore, section 6.1 illustrates potential data types which could be used. The second dimension to distinguish service analytics is based on different levels of maturity of applied methods, from basic analytics (reports and queries) and advanced analytics (statistical analysis and data mining). Section 6.2 therefore illustrates a method of applying advanced analytics which could be used for service costing.

\subsection{Mapping Data Sources to Customer Factors by Means of USDL}

We propose a semantic layer on top of the different data sources, which enables the mapping of data sources to customer factors, by specifying the calculation of customer metrics which refer to customer attributes.

- Co-Creation $\left(C_{i}\right)$ : A good source for deriving metrics for co-creation would be historical encounter data, containing information on the past interaction between the customer and the provider organization through different channels. Using USDL, this interaction data can be analysed in order to derive meaningful metrics for interaction, such as analysing participants and their roles in meetings.

- Information $\left(I_{i}\right)$ : For deriving metrics related to customer information, analysing documents of past customer projects could be helpful. Using USDL, it would be possible to identify the documents required in each step of the process. Afterwards the quality of these documents could be benchmarked and compared to the average quality.

- Technology $\left(T_{i}\right)$ : Using USDL, which allows modelling technology as a resource, enables an analysis of technology involvement in the past customer projects. A potential data source to investigate would be vouchers of invoices, if the usage of specific technology has been billed individually in the past.

- Experience $\left(E_{i}\right)$ : As USDL allows modelling individual participants, the experience of each involved human resource in a specific point of the service process would be possible. A good data source would be a time tracking database, where employees track their specific activities for a customer project.

\subsection{Applying Analytics}

We propose using data mining for predicting customer attributes and finally estimating service costs. Data mining targets at discovering patterns in data in order to derive predictions [19]. The input for running data mining algorithms is a set of instances. Each instance consists of values of a fixed, predetermined set of features, called attributes. An algorithm is then applied to the set of instances to discover 
patterns and structures that can be used to make predictions about unknown data. In our case, we would analyse data from past maintenance services and services provided to the same customer, of which we want to forecast service costs. The calculated metrics and historical service costing results would serve as our attributes. Forecasting costs requires the prediction of a numeric quantity. However, we cannot assume a linear correlation between calculated metrics and costs. Therefore, nonlinear methods are required. Potential algorithms are e.g. Neural Networks, Polynomial Regression, or k-Nearest-Neighbour. The derived models are then applied to new cases where service costs need to be estimated.

\subsection{Example of Customer Factor Calculation}

Based on input from the customer care database, we can define nine different customer attributes (three for each customer factor). Examples for this nine attributes are (1) the quality of the problem description for $I_{l},(2)$ the number of required diagnostic devices for $T_{1}$, and (3) the experience of the contact person for $E_{l}$. For each customer attribute we have used an individual metric, e.g. the length of a problem description for (1). With a forecasted weighing $\left(I_{w}=0.4, T_{w}=0.25, E_{w}=0.35\right)$ and a $C_{l}=3.33$, we obtain customer factor values for ten different co-creation instances $\left(C C_{i}\right)$ of $(43,43,40,40,30,33,30,30,30,30)$. From these results, we can conclude a varying customer involvement from $\mathrm{CC}_{1}$ to $\mathrm{CC}_{6}$ and stabilization from $\mathrm{CC}_{7}$ to $\mathrm{CC}_{10}$. The decreasing customer factors suggest a decreasing customer attribute quality with negative consequences to reach forecasted service costs. To identify the cause of change a drill down to individual customer attribute values is possible and necessary.

\section{Conclusions}

From provider side service costing needs more attention. Since current models, such as Activity-Based Costing (ABC), lack accuracy when applied to services and $A B C$ ignores the central aspect of co-creation. Our work tries to overcome these problems using the concept of customer factors to decrease uncertainty about service performance. With our customer factors (co-creation, information, technology and experience) we are able to measure a customer's individual impact on co-creation. Customer factors are integrated into our three layered approach using customer attributes, customer factors and Time-Driven Activity-Based Costing. Another aspect which has not been explored, is the development of a systematic approach to analyse service systems. In this field, we proposed an abstraction layer (by relying on Linked USDL) which mediates the data sources and enterprise information systems which store service instance data. This layer serves as a conceptual model which simplifies the development and reuse of algorithms for service analytics. In the future, we will exploit the potential of service performance measurement by combining our proposed approach with methods from operations research. 


\section{References}

[1] Spohrer, J., Vargo, S., Caswell, N., Maglio: The Service System is the Basic Abstraction of Service Science. In: 41st Hawaii International Conference on System Sciences, Hawaii (2008)

[2] Erkoyuncu, J.A., Roy, R., Shehab, E., Cheruvu, K.: Understanding service uncertainties in industrial product-service system cost estimation. The Int. J. of Advanced Manufacturing Technology 52(9-12) (2010)

[3] Cooper, R., Kaplan, R.S.: Measure Costs Right: Make the Right Decisions. In: HRB (1988)

[4] Anderson, S.R., Kaplan, R.S.: Time-Driven Activity-Based Costing. Mcgraw-Hill (2007)

[5] Flandel, G., Blaga, S.: Elements of the production of services. In: Modern Concepts of the Theory of the Firm - Managing Enterprises of the New Economy, pp. 175-189. Springer, Berlin (2004)

[6] Frisch, R.: Theory of Production, pp. 3-5. Springer (2009)

[7] And de Vries, W., Kasper, H., van Helsdingen, P.: Service Marketing Management - An International Perpective, pp. 8-20. John Wiley \& Sons, England (1999)

[8] Bergholtz, M., Johannesson, P., Andersson, B.: Towards a model of services based on cocreation, abstraction and restriction. In: Jeusfeld, M., Delcambre, L., Ling, T.-W. (eds.) ER 2011. LNCS, vol. 6998, pp. 476-485. Springer, Heidelberg (2011)

[9] Moll, J.: The patient as service co-creator. In: Proceedings of the 11th Biennial Participatory Design Conference (PDC 2010). ACM, USA (2010)

[10] Sioukas, A.V.: User involvement for effective customization: An empirical study on voice networks. IEEE Transactions on Engineering Management 42(1), 39-49 (1995)

[11] Lu, Y., Wei, H.: Research on the motivation of the customer participation based on gray relational analysis. In: International Conference on Business Management and Electronic Information (BMEI), pp. 438-441 (2011)

[12] Zhang, X., Wang, G.-X., ShangGuan, J., Xiao, Y.: Value co-creation with customers and its impact on capabilities. In: IEEE International Conference on Industrial Engineering and Engineering Management (2008)

[13] Furstner, I., Anisic, Z.: A possibility for optimal customer profile definition. In: 11th International Symposium on Computational Intelligence and Informatics (CINTI) (2010)

[14] Dorn, J., Seiringer, W.: A Prototype for Service-Based Costing. In: 46th Hawaii International Conference on System Sciences (HICSS), Hawaii (2013)

[15] Cardoso, J., Barros, A., May, N., Kylau, U.: Towards a Unified Service Description Language for the Internet of Services: Requirements and First Developments. In: IEEE International Conference on Services Computing. IEEE Computer Society Press, USA (2010)

[16] Shostack, G.L.: How to design a service. European J. of Marketing 16 (1982)

[17] Grüninger, M., Fox, M.S.: The role of competency questions in enterprise engineering. In: Proceedings of the IFIP WG5, vol. 7, pp. 212-221 (1994)

[18] Fromm, H., Habryn, F., Satzger, G.: Service Analytics: Leveraging Data Across Enterprise Boundaries for Competitive Advantage. In: Globalization of Professional Services, pp. 139-149 (2012)

[19] Witten, I.H., Frank, E.: Data Mining: Practical machine learning tools and techniques (2005) 